Send your letters to the Editor,

British Dental Journal,

64 Wimpole Street

London

W1G 8YS

E-mail bdj@bda.org

Priority will be given to letters less than 500 words long.

Authors must sign the letter, which

may be edited for reasons of space.

\section{MISSING THE CRUX}

Sir, I would like to challenge some of the statements made by Tomás et al. Confirm the efficacy (BDJ 2008; 205: 3). Given the problems with previously published guidelines on endocarditis prophylaxis where cardiac lesions were stratified according to risk, it was decided within the NICE Guideline Development Group (GDG) not to stratify risk groups. Hence in the NICE guideline we simply stated those at risk and those not at risk (isolated atrial septal defect, repaired patent ductus arteriosus and repaired ventricular septal defects). From available evidence, it was concluded that those who have endocarditis have high rates of mortality and those with prosthetic valve endocarditis have a higher rate of mortality compared with native valve endocarditis. The message we were trying to portray was that all patients with known risk factors are at risk of developing endocarditis and should be regarded as such by dentists; this guideline does not change that.

There is no doubt that antibiotics reduce levels of bacteraemias or else we would not prescribe them therapeutically, but with a single dose they do not universally eliminate bacteraemia. There remains no evidence that antibiotics are either effective or ineffective in preventing endocarditis.

What Tomas et al. are missing is the main crux of the evidence and one of the pivotal factors which led the GDG to reach the published conclusions, namely that bacteraemia occurs following everyday activities which are never covered with prophylactic antibiotics. Although evidence was not found, it is likely that everyday activities other than those involving the oral mucosa produce a bacteraemia. Therefore, admittedly there is no evidence to support the statement but in those patients at risk of developing endocarditis achieving and maintaining a high level of oral health should be the aim of dentists as it is for all our patients.

Whilst there are universal calls to increase the evidence base to inform our clinical decisions in this field, I remain sceptical that this is feasible or likely in the short term since the development of endocarditis is relatively rare compared to the large number of bacteraemias that are occurring on a daily basis. Crude evidence will come from the Hospital Episode Statistics whereby the diagnosis of all patients admitted to hospital is recorded; if a significant rise in cases of endocarditis is recorded in the light of the NICE guidance then an urgent rethink will have to take place.

In the meantime, as a profession, dentists must ensure they continue to identify patients at risk of developing endocarditis, ensure high levels of oral health are achieved in these patients and ensure patients are aware of the signs and symptoms of endocarditis to enable prompt investigation and treatment if required.

R. Oliver, Manchester

DOI: 10.1038/sj.bdj.2008.851

\section{MAGIC WAND}

Sir, the experiences summarised in the paper by Versloot et al. (BDJ 2008; 205: E2) do not reflect our experience in NHS Highland.

I have been using The Wand for over two years in my practice as senior dentist with special interest in children's dentistry. I see a large number of patients referred into the anxious child service whose own dentists have been unable to provide treatment under local anaesthetic despite best efforts. Previously, a mixture of behaviour management, nitrous oxide sedation and general anaesthesia (GA) has been used. Many of these children can be acclimatised very quickly with The Wand to tolerate treatment with local anaesthesia. It has made treating the very anxious children who are referred to us a much quicker and simpler process, reducing the reliance on sedation and GA.

The Wand allows the administration of very comfortable infiltrations (buccal, palatal and intra-ligamental) and ID blocks with a much less threatening appearance than a traditional dental syringe. Lieberman ${ }^{1}$ expressed the importance of the change in patient perception: 'Since The Wand is so unique in appearance, the patients do not relate it to their previous experiences or preconceived ideas. It has been our experience that an overwhelming percentage of patients who verbally express fear of the "shot", seem greatly reassured that we will use The Wand instead.'

Held in a pen grip, the approach to the patient is much more relaxed, particularly when it is reduced in size by snapping the handle to its shorter length and it is also significantly less strain for the operator than giving a slow, controlled injection with a conventional syringe. The shape of the needle allows very easy introduction into tissue and the use of a pre-puncture technique to ensure comfortable penetration. The reliability of inferior dental blocks is improved due to the ease of rotation of the wand using the bi-rotational injection technique, reducing needle deflection as it passes through tissue. ${ }^{2}$

Performing pain free palatal infiltrations quickly and easily using a prepuncture technique enables orthodontic extractions with much less anxiety and comfortable PASA and AMSA techniques produce reliable anaesthesia in 
the maxilla with a greatly reduced dose and minimal labial anaesthesia.

A significant advantage of The Wand is the very positive, reliable, simple aspiration facility. The cross infection control is simple and the re-sheathing is very effective, reducing the possibility of needlestick injury. It has proved very cost effective in reducing reliance on sedation and GA.

We use two Wands which are in daily use for a large number of procedures. After two years we have suffered no reliability problems with little maintenance beyond periodic lubrication. There is a learning curve and a shift in perception when first using the instrument and appropriate behaviour and anxiety management are still the cornerstone of treating children. However, my colleague in the department is a vocational trainer and we have trained a number of FY2 dentists in its use. Their feedback has been very positive.

In the words of one (formerly anxious) young patient: 'It's great now you don't need to get the jag'!

G. Jackson, Inverness

1. Lieberman W H. The Wand. Pediatr Dent 1999 . 21: 124

2. Malamed SE. Handbook of local anesthesia, $5^{\text {th }}$ ed. Mosby, 2004.

DOI: $10.1038 /$ sj.bdj.2008.852

\section{BETWEEN TWO STOOLS}

Sir, the adage 'if it works don't fix it', could have been applied to the system of remuneration to dentists prior to 2006.

From 1948 till 1990 a fee per item of service was extant as the method of payment for dentists working in the NHS. The change to a capitation payment system primarily for children was due to the fall in incidence of dental disease and the increasing provision for preventative dentistry.

But if you were paid for saying 'hello' to the patient or for just getting a form signed, it did not take too long to note that the art of supervised negligence reared its ugly head by those very few dentists (probably less than 0.5\%), and that as usual the Department of Health prevaricated in changing the system long after the aberration in the payment system became obvious.

The dental advisors at the Dental Practice Board informed the Department of Health as soon as it became apparent that there were distortions in the new system, and the way to correct these. But of course the professionals at the Dental Practice Board were not listened to, and another crisis involving adjustments (reduced earning!) put another nail in the coffin for an equitable system for the patient, and the morale of the dentist was dealt another blow.

The new system of UDA (Units of Dental Activity) was supposed to create a system which ironed out the problems of the item of service vis-à-vis capitation, but like the veritable clown it fell between two stools.

It is too early to say whether an improvement in oral health is being sustained, but there is a reduction in the availability of treatment within the NHS, a burgeoning and very expensive private sector, and a demoralised dental profession.

It is possible that the new system is a halfway house to the privatisation of dentistry (compare this history to that of the optical services), but if so, noone seems to have considered alternative methods of the delivery of payment for treatments such as grant in aid or 'mutual' ie co-operative insurance systems, designed and managed by the dental profession, with some support from central government funding.

S. Barsam, by email DOI: 10.1038/sj.bdj.2008.853

\section{CONSENT AND CAPACITY}

Sir, we were interested to read the article on consent and capacity ${ }^{1}$ which gave a helpful insight into the implications of the Mental Capacity Act for special care dentistry. However, we would like to clarify several points within the article which may have caused confusion for readers.

The suggestion that implied consent for both examination and treatment, which happens when patients sit in a dental chair and voluntarily open their mouth, is acceptable, is not correct. Consent to a dental examination can be implied in this circumstance, but expressed, informed consent is required for all treatment, including the taking of radiographs. The quality of the explanations and information given to (and understood by) the patient are crucial to obtaining informed consent.

In addition, while the DDU would agree that a patient's signature on a consent form may help to confirm that consent has been obtained, it cannot be relied upon on its own, as suggested in the article. A patient's signature on a treatment plan or consent form is in our experience of secondary significance to the notes made in the clinical records of conversations where the patient is given explanations and warnings, particularly concerning treatment options and potential complications.

In some cases a patient's condition may change between the initial consultation and the time at which treatment takes place, which can affect the nature of treatment and its likely risks and chances of success. In such cases the dental professional will need to explain the changes and to obtain consent again. Re-confirmation of consent immediately before treatment is always prudent, as of course is making a record of this.

If the treatment is complex, has particular risks, or several treatment options are available and have been discussed, then the DDU recommends that this is noted into the patient's records. If an allegation of lack of consent is made, entries in clinical records are more valuable from an evidential standpoint than a signature on a consent form. The DDU does not, however, discourage the use of consent forms, written treatment plans and explanatory leaflets.

Finally, while the article is mainly about adults without capacity, the authors state that informed consent is required from all patients or, in the case of children, parents'. However, children under 16 can consent to treatment if they understand its nature, purpose and hazards following review by the House of Lords in the 1985 Gillick judgement.

To be able to consent, the child must understand the nature of the proposed treatment and fully understand and appreciate the consequences of the treatment, the alternatives and the failure to treat. It is only where a child is not Gillick competent, that whoever has parental responsibility may give authority to treat under the Children Act 1989.

R. Hoppenbrouwers, Head of the DDU

1. Dougall A, Fiske J. Access to special care dentistry, part 3. Consent and capacity. Br Dent J 2008; 205: 71-81.

DOI: $10.1038 /$ sj.bdj.2008.854 